\title{
Ultrasonic Enhanced treatment wastewater dairy farming organics of single-factor test
}

\author{
Yong-guang Bi1,2,, Kuan-xin Zhang 2 \\ 1 Key Laboratory for Agricultural Environment, Ministry of Agriculture, \\ P.R.China, 100081 \\ 2 College of Pharmacy, Guangdong Pharmaceutical University, \\ Guangzhou 510006, Guangdong, China \\ E-mail:biyongguang2002@163.com
}

\begin{abstract}
In dairy farming simulated wastewater resorcinol as the research object, examines the ultrasound enhanced treatment of resorcinol single factor test, the results showed that: resorcinol degradation rate increased with the ultrasonic power is reduced, when sonication is $200 \mathrm{~W}$, its degradation rate of $45 \%$; resorcinol degradation rate with ultrasound increases the temperature rises, when the ultrasound when the temperature is $60^{\circ} \mathrm{C}$, the degradation rate was $45.3 \%$; resorcinol the degradation rate increases as ultrasonic time increases, when the temperature of the ultrasonic $60 \mathrm{~min}$, its degradation rate of $47.1 \%$; studies show that ultrasound is a better method for degradation of organic pollutants in new resorcinol, and to provide a basis for follow-up research process optimization.

Keywords: ultrasound; cows wastewater; resorcinol; single factor; test
\end{abstract}

\section{Introduction}

Environmental pollution caused by livestock industry include water pollution, air pollution and soil pollution, and environmental degradation directly affects the health status of the farm itself, reducing the ability of animal epidemic prevention, endangering their lives, reducing the quality of animal products, indirectly affecting human health hazard, thereby affecting the sustained and healthy development of animal husbandry. According to the first national census of pollution sources to grasp the situation of agricultural emissions sources, agricultural sources of emissions greater impact on the water environment. In agricultural sources of pollution, the more prominent is the livestock industry pollution, chemical oxygen demand of the livestock industry, total nitrogen and total phosphorus emissions accounted for 99\% of agricultural sources, respectively, $42 \%$ and $62 \%$. To fundamentally solve the problem of water pollution, agricultural sources of pollution prevention and control shall incorporate important agenda of environmental protection, while increasing emissions of pollutants "Twelve Five" period of ammonia to binding targets, which for livestock and poultry manure sewage discharge made more stringent 
environmental requirements. Aquaculture wastewater with a typical "three high" characteristics, high COD, high ammonia, high-SS, while aquaculture is Boli industry, can not afford the high cost of sewage treatment, the current treatment process to deal with organics Lord, to get a more COD substantial reductions, but not enough to reduce the amount of ammonia is difficult to achieve the required emissions of ammonia treatment so there are certain technical and economic difficulty ${ }^{[1]}$.

Due to the high ammonia content of dairy wastewater, often leading biochemical process carbon is not enough, $\mathrm{C} / \mathrm{N}$ is too low, thus affecting the removal of total nitrogen, carbon will increase if additional processing costs, while the pig industry a traditional industry wastewater treatment for limited funds, so the pig farm wastewater ammonia standard is very difficult. Before the biochemical processing in order to reduce the difficulty of subsequent biological denitrification process, is a new way of swine wastewater treatment. Currently pig farm wastewater treatment technologies have materialized ammonia, biological, and ecological treatment method in practical engineering, these methods need to be used in combination to achieve the effluent of each pollutant emission standards.

Generally refers to the frequency of the ultrasonic 20-200kHZ sound waves. When the sound intensity increases to a certain amount, it will produce its influence spread in the media, so that the state of the medium, composition, function and structure changes, collectively referred to as ultrasound effect. When the ultrasonic wave propagation in the medium, due to the interaction of ultrasound with the media, the media physically and chemical changes, resulting in a series of mechanical, thermal, and electromagnetics, and chemical effects of ultrasound, including the following four kinds of effects : (1)cavitation. Most sonicated in liquid cavitation and related technologies; (2)mechanical effects. Mechanical action may contribute to the ultrasonic emulsification of the liquid, gel and solid dispersion of the liquefied; (3) thermal effects. Since the high frequency ultrasonic waves, energy, can produce a significant effect when the heat absorbing medium; (4)chemical effects. The role of ultrasound can induce or accelerate certain chemical reactions occur ${ }^{[2]}$.

Because ultrasound is a set of advanced oxidation technology, a variety of water treatment incineration, supercritical water oxidation technology in one, can improve the reaction conditions, to accelerate the reaction rate and improve the reaction yield, but also to make certain chemical reactions can be difficult to carry out successfully carried out, and gradually developed into a new water treatment technology. To the field of water treatment for refractory toxic organic compounds and COD can achieve better treatment effect, many researchers ultrasonic and other technical processes used in combination.

In this paper, for the current dairy farming biodegradable organics in wastewater problems, dairy farming research sonication process wastewater resorcinol, are provided for the ultrasound and other processing techniques combined with the reactor design, scale, provide a theoretical basis for industrialization . 


\section{Materials and methods[3]}

\subsection{Materials and Reagents}

Resorcinol, anhydrous sodium carbonate, sodium thiosulfate, soluble starch, hydrochloric acid, potassium iodide, potassium bromide, potassium bromide, potassium dichromate, sodium hydroxide, sulfuric acid, hydrogen peroxide, sodium chloride, these reagents were analyzed pure reagents.

\subsection{Instruments and Equipment}

UV1101 UV/ visible spectrophotometer (Shanghai Tian Mei Scientific Instrument Co., Ltd.); AY120 electronic analytical balance (Shimadzu Corporation); KH-400KDB CNC high-power ultrasonic cleaner (Kunshan Ultrasonic Instrument Co., Ltd. Wo Chong).

\section{3 preparation of the test solution}

\subsection{Standard curve and degradation rate calculation method[3]}

\section{Results and Discussion}

\subsection{Examine the impact on the degradation rate of ultrasonic power}

Take stock solution of resorcinol 30mL6 parts, the time set for $20 \mathrm{~min}$, at room temperature, inspection ultrasonic power 200W, 240W, 280W, 320W, 360W, $400 \mathrm{~W}$ influence on the degradation rate of resorcinol. The results are shown (Fig.1).

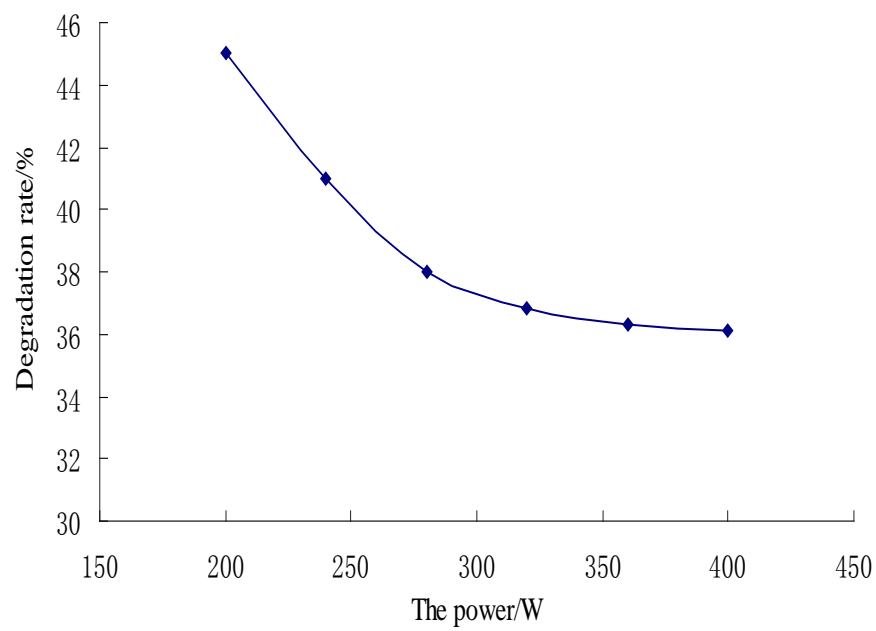

Fig.1 Effect of ultrasonic power on the degradation rate of resorcinol Some scholars believe that the degradation rate of organic matter simply 
repeating a strong increase in the presence of a great value, when more than this value, the degradation rate of simply repeating the strong increases. Found in this experiment combined with power $200 \mathrm{~W}$ power up after ultrasonic treatment reduced the ability of resorcinol. The reason for this phenomenon is the main mechanism of the degradation of organic matter and ultrasound. Power sources ultrasonic degradation of organic matter is acoustic cavitation, there are more cavitation bubbles when the system will be beneficial to the degradation. As the power increases, the number of cavitation bubbles rising, when the power is too high, small cavitation bubbles collide more intense, and combined with each other to form large bubbles floating to surface, such big bubbles will weaken sound effects. While the lower portion of the acoustic transmitter to generate a large number of holes leading to the acoustic transmitter is not sufficiently in contact with the reaction liquid, cavitation bubbles are formed in the negative phase of the sound of the acoustic shield, so that the sound field energy can be utilized to reduce, not to be conducive to acoustic reaction . From the above analysis and the results should be used in ultrasonic power of $200 \mathrm{~W}$.

\subsection{Investigate the influence of temperature on the degradation rate of ultrasound}

Take stock solution of resorcinol 30mL5 parts, under the conditions set ultrasonic power $200 \mathrm{~W}$, the time is set to $20 \mathrm{~min}$, investigate ultrasonic temperature $20^{\circ} \mathrm{C}, 30^{\circ} \mathrm{C}, 40^{\circ} \mathrm{C}, 50^{\circ} \mathrm{C}, 60^{\circ} \mathrm{C}$ for resorcinol degradation rate impact. The results are shown (Fig.2).

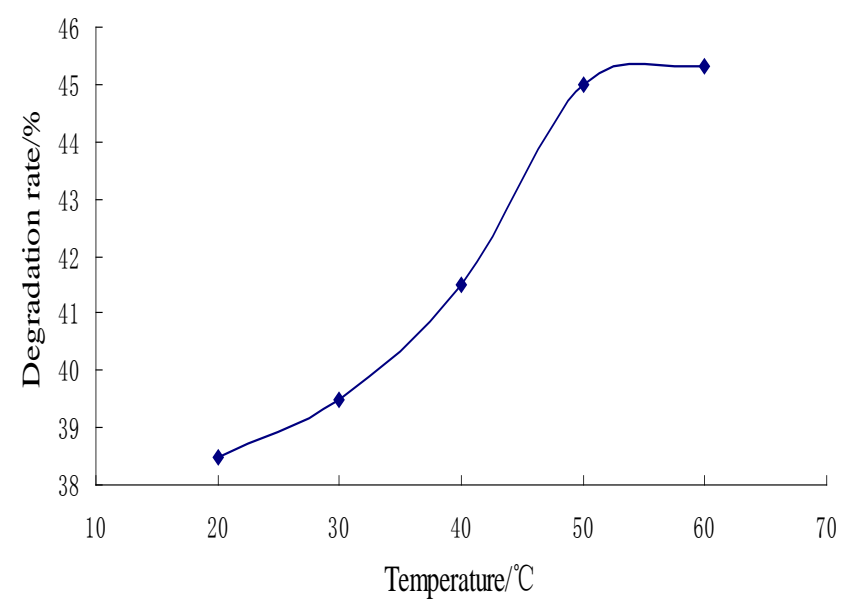

Fig.2 Ultrasound temperature on the degradation rate of resorcinol

As can be seen from Fig.2, the increase in the degradation temperature of the resorcinol help, but when the temperature rises to a certain extent, to improve the obvious degradation. According to the principle of ultrasonic degradation of organic contaminants, polar, non-volatile substances, mainly degradation by 
oxidation. Instantaneous collapse of cavitation bubbles, steam bubbles inside the thermal decomposition to produce a series of reactions, radical ion produced has a strong oxidation resistance, high temperature and pressure conditions can decompose organic pollutants. The temperature rises to a certain degree, as the temperature rises, the solubility of gases decreases, the surface tension decreases, the saturation vapor pressure increases, the ultrasonic cavitation negative, so the degradation rate is no longer increased. And the results can be seen from the above analysis should be used ultrasound temperature $50^{\circ} \mathrm{C}$.

\subsection{Investigate the influence of ultrasonic time on the degradation rate}

Take stock solution 30mL5 parts of resorcinol, ultrasonic power 200W under set conditions, the temperature set at $50^{\circ} \mathrm{C}$, ultrasonic inspection time $20 \mathrm{~min}$, $30 \mathrm{~min}$, 40min, 50min, 60min for resorcinol degradation rate impact. The results are shown (Fig.3).

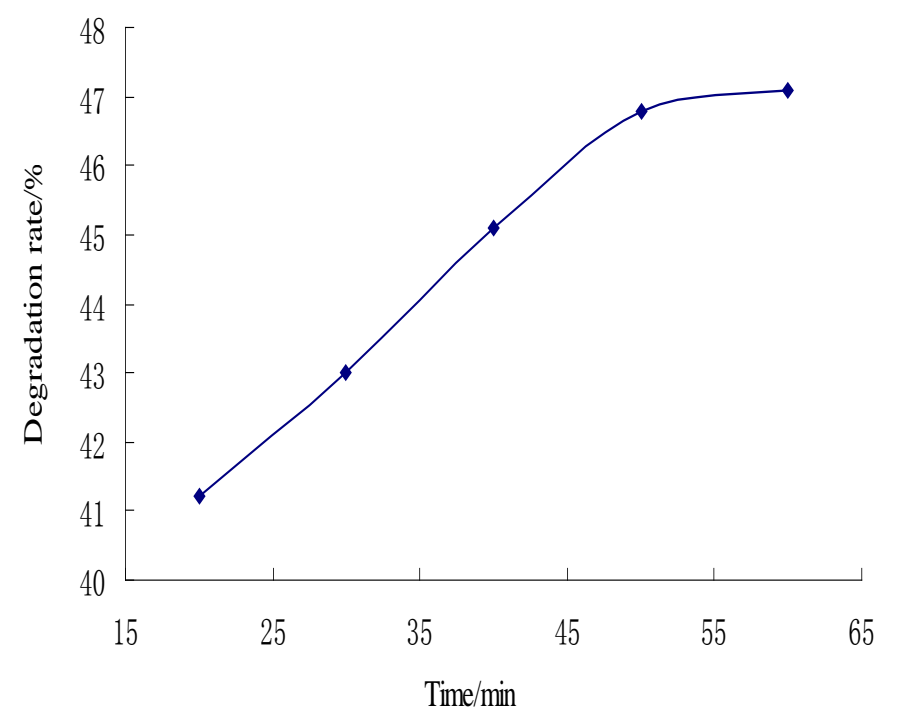

Fig.3 Ultrasonic time impact on the degradation rate of resorcinol

Degradation results from resorcinol (Fig.3) shows that with time, resorcinol degradation rate gradually increased, reaching a peak $(46.84 \%)$ in $50 \mathrm{~min}$ time. Additional time, the extraction rate increased marginally. According to radical reaction theory, high temperature cavitation in produced under high pressure, the water molecules cleaved to generate $\cdot \mathrm{OH}$ radicals and $\cdot \mathrm{H}$ radicals, an appropriate extension of ultrasonic time, a large number of $\bullet \mathrm{OH}$ radicals are very reactive organic matter in water completely oxidized to $\mathrm{CO}_{2}$ and $\mathrm{H}_{2} \mathrm{O}$. With time, this water should be free radicals or increase of the charged ions, the radical identical to each other but also increases the chance of composite, less fine bubbles, so that the degradation rate was not significantly increased. 


\section{Conclusion}

By exploring the ultrasonic power, the impact of time, temperature, and other factors on resorcinol degradation rate, results showed that: resorcinol degradation rate increased with the ultrasonic power is reduced when the sonication is $200 \mathrm{~W}$, its degradation rate of $45 \%$; resorcinol degradation rate increases with increasing temperature, ultrasound, ultrasound when the temperature is $60^{\circ} \mathrm{C}$, its degradation rate of $45.3 \%$; resorcinol degradation rate with ultrasound time increases, the time when the ultrasonic temperature for $60 \mathrm{~min}$, the degradation rate was $47.1 \%$; studies show that ultrasound is a new method better resorcinol degradation of organic pollutants and to provide for the subsequent process optimization research base.

\section{Acknowledgements}

This work was financially supported by the Open Fund of Key Laboratory for Agricultural Environment, Ministry of Agriculture, P.R.China

\section{References}

[1]Komegay ET and A F Harper.Environmental nutrition:Nutrient management Strategies to reduce nutrient exeretion of swine water.The Professional Animal Seientist.1997.13:99-111

[2]National Researeh couneil, Nutrient Requirements of swine [M].Tenth reviseded. Washington, DC:National Aeademy Press,1998

[3]Yong-guang Bi, Kuan-xin Zhang.Dairy farming simulated organic wastewater of degradation Experiment by hydrogen peroxide[J]. Advanced Materials Research, 2014, (1015):729-732. 